Address for Correspondence: Prof. Zhiyuan Hu, CAS Key Laboratory of Standardization and Measurement for Nanotechnology, CAS Center for Excellence in Nanoscience, National Center for Nanoscience and Technology, Beijing 100190, China.

Email: huzy@nanopep.com

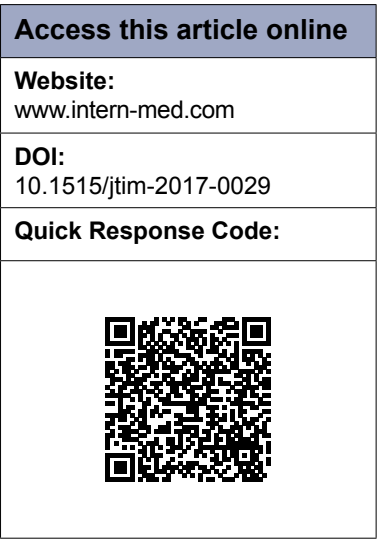

\title{
The functions of circulating tumor cells in early diagnosis and surveillance during cancer advancement
}

\author{
Yuqiao Wang ${ }^{1}$, Ying Zhou ${ }^{2}$, Zhiyuan $\mathrm{Hu}^{2,3}$ \\ ${ }^{1}$ The Cooper Union for the Advancement of Science and Art, New York, USA; \\ ${ }^{2}$ Center for Neuroscience Research, School of Basic Medical Sciences, \\ Fujian Medical University, Fuzhou, Fujian Province, China; \\ ${ }^{3}$ CAS Key Laboratory of Standardization and Measurement for Nanotechnology, CAS Center \\ for Excellence in Nanoscience, National Center for Nanoscience and Technology, Beijing, China
}

\section{INTRODUCTION}

Circulating tumor cells (CTCs) are the debris generated from a cluster of primary or metastatic tumor cells at a certain organ. In 1869, the Australian researcher Thomas Ashworth first discovered a pack of CTCs in the blood test from a metastatic cancer case. ${ }^{[1]}$ In 1976, Nowell amended the definition of CTC as tumor cells derived from primary tumors or metastatic tumors with the ability to get out of the basement membrane and invade into the blood vessels through the tissue matrix. ${ }^{[2]}$ Currently, CTCs are referred to various types of tumor cells in the peripheral blood (PB) in general. In this case, a "mother tumor" released some minor carcinoma cells that shared exactly the same characters as those of the mother tumor according to the blood test. ${ }^{[1]}$ Considering the absence of adherent fibril proteins and a smaller size relative to primary carcinoma cells, CTCs have a considerably great mobility in bloodstream. ${ }^{[3]}$ Such a mobility is believed to result in the metastasis of cancer and the deprivation of nutrients to normal cells, which give rise to the major cancer-related deaths.

CTCs detected in PB of patients with cancer can be identified as isolated CTCs and circulating tumor microemboli (CTM). ${ }^{[4]}$ Tumor cells lose their cell-to-cell adhesion and undergo epithelial-mesenchymal transition in order to enter blood circulation. ${ }^{[5]}$

It is well known that cancers detected at an advanced stage remain largely untreatable. The liquid biopsy involving CTC enumeration is necessary by taking a few milliliters of blood from the patient and counting the number of CTCs per unit volume. Provided the CTCs are the fragments of primary tumor cells, it can be concluded whether these fragments captured from the blood sample display any characters of the primary tumor cell. The diagnosis of cancer will then be carried out by sequencing the DNA/RNA extracted from the CTCs, usually via NGS (next-generation sequencing). ${ }^{[6]}$ As the liquid biopsy is a non-invasive inexpensive blood test, multiple samples can be easily obtained to keep track of the development of cancers. The bottleneck of current CTC study and application mainly focus on integrating a rapid CTC enumeration with a highly personalized assay panel.

\section{CURRENT STATUS}

\section{Recent process of CTC detection}

Currently, the CTC-oriented early detection of cancers, usually known as "liquid biopsy," addresses the collection tools of CTCs and the clinical application of monitoring the extent of tumor metastasis. Considering the abundance of blood sample, less unnecessary pain created during sample acquirement, and higher sensitivity of detection during the assay, determining the level of CTCs or the free circulating tumor DNA (ctDNA) released in the patients' bloodstream is easy to be performed and repeated over time. Recently, a series of primary studies carried out in laboratories 
have been reported as valid and effective steps to proceed to further clinical applications.

\section{Application of CTCs in breast cancer diagnosis}

In March 2013, Dawson et al. revealed a clear relevance between the concentration of CTCs and expected lifetime as well as the comparison between the sensitivity of three different biomarkers in diagnosis of breast cancer: Cancer Antigen 15-3 (CA 15-3), cell-free ctDNA, and CTCs. ${ }^{[7]}$ In this study, a targeted or whole-genome sequencing to track alternation in somatic genome scheme was designed and performed; personalized assays was set to quantify in vitro circulating tumor DNA, CA 15-3 levels and numbers of circulating tumor cells simultaneously. As a result, ctDNA was successfully observed in 29 of 30 women (97\%) with somatic genomic alterations identified. CA 15-3 and CTCs were detected in 21 of 27 women $(78 \%)$ and 26 of 30 women $(87 \%)$, respectively. ${ }^{[7]}$ In each $7.5 \mathrm{~mL}$ of blood test sample, subject to four different quantiles of ctDNA detected, the probability of a patient's expected survival is roughly indirectly proportional to overall survival (OS) in days, which is consistent with the concentration of cellfree ctDNA, and verifies the function of ctDNA level in indicating the extent of tumor metastasis advancement.

In 2004, Cristofanilli et al. tested 177 patients with measurable metastatic breast cancer (MBC) for levels of CTCs both before the patients were to start a new line of treatment and at the first follow-up visit, determined with the use of standard imaging studies at the participating centers. ${ }^{[8]}$ They found that patients in a training set with levels of CTCs equal to or higher than 5 per $7.5 \mathrm{~mL}$ of whole blood, as compared with the group with fewer than 5 CTCs per $7.5 \mathrm{~mL}$, had a shorter median progression-free survival (PFS; 2.7 months vs. 7.0 months, $P<0.001$ ) and shorter OS (10.1 months vs. $>18$ months, $P<0.001) .{ }^{[8]}$ The number of CTCs before treatment is proved to be an independent predictor of PFS and OS in patients with MBC. ${ }^{[8]}$

\section{Application of CTCs in colorectal cancer diagnosis}

In 2008, Cohen et al. enumerated CTCs in the PB of 430 patients with metastatic colorectal cancer (mCRC) at baseline and after starting first-, second-, or third-line therapy and measured CTCs using an immunomagnetic separation technique. ${ }^{[9]}$ They found that patients with unfavorable compared with favorable baseline CTCs had shorter median PFS (4.5 months vs. 7.9 months; $P=0.0002)$ and OS (9.4 months vs. 18.5 months; $P<0.0001$ ), showing that the number of CTCs before and during the treatment is an independent predictor of PFS and OS in patients with mCRC..$^{[0]}$

\section{Application of CTCs in castration-resistant prostate cancer diagnosis}

To establish the relationship between posttreatment CTC count and OS in castration-resistant prostate cancer (CRPC), blood was drawn from patients with CRPC with progressive disease starting a new line of chemotherapy before treatment and monthly thereafter, and patients were stratified into predetermined favorable or unfavorable groups $(<5$ and $\geq 5$ CTC $/ 7.5 \mathrm{~mL}) \cdot{ }^{[10]}$ De Bono et al. (2008) found that patients with unfavorable pretreatment CTC (57\%) had shorter OS (median OS, 11.5 vs. 21.7 months; Cox hazard ratio, 3.3; $P<0.0001$ ), indicating that CTCs are the most accurate and independent predictor of OS in CRPC. ${ }^{[10]}$ In addition, these data led to Food and Drug Administration clearance of this assay for the evaluation of CRPC. ${ }^{[10]}$

\section{Circulating tumor cell clusters}

CTC clusters are present in the blood of patients with cancer but their contribution to metastasis is not well defined. Using mouse models with tagged mammary tumors, Aceto et al. (2014) demonstrated that CTC clusters arise from oligoclonal tumor cell groupings and not from intravascular aggregation events. ${ }^{[1]}$ Although rare in the circulation compared with single CTCs, CTC clusters have 23- to 50-fold increased metastatic potential. In patients with breast cancer, single-cell resolution RNA sequencing of CTC clusters and single CTCs, matched within individual blood samples, identifies the cell junction component plakoglobin as highly differentially expressed. ${ }^{[1]}$ In mouse models, knockdown of plakoglobin abrogates CTC cluster formation and suppresses lung metastases. ${ }^{[1]}$ In patients with breast cancer, both the abundance of CTC clusters and the high tumor plakoglobin levels denote adverse outcomes. ${ }^{[1]]}$ Thus, CTC clusters are derived from multicellular groupings of primary tumor cells held together through plakoglobin-dependent intercellular adhesion, and though rare, they greatly contribute to the metastatic spread of cancer. ${ }^{[1]]}$

\section{Relevance of CTC concentration and patients' prognosis}

A CTC assay is a powerful tool not only when it works alone but also when it couples with chemotherapy, in which the concentration of CTCs serves as a reference to assess the effects of medications. In September 2015, Alva et al. explored the application of CTCs analysis in evaluating the outcome of neoadjuvant chemotherapy using cisplatin by recruiting patients undergoing neoadjuvant chemotherapy, patients undergoing tumor metastasis, and healthy adult volunteers and determining the CTC concentration on the course of treatment at different phases. ${ }^{[12]}$ In the article "Circulating Tumor Cells as Potential Biomarkers in 
Bladder Cancer," Alva et al. divided 30 human volunteers into neoadjuvant group, metastatic group, and healthy group. The blood samples of the volunteers were then analyzed using the IsoFlux CTC Enrichment Kit, which was a selection apparatus based on the principles of negative enrichment by a CD45-negative and cytokeratin-positive filtrator. Upon the ultimate CTC assay through NGS, the number of CTCs declined viably. ${ }^{[12]}$

Despite progressive improvements in the management of patients with locoregionally confined, advanced-stage solid tumors, distant metastasis remains a very common - and usually fatal - mode of failure after attempted curative treatment. ${ }^{[13]}$ Surgery and radiotherapy are the primary curative modalities for these patients, often combined with each other and/or with chemotherapy. ${ }^{[13]}$ Surgical interventions in patients with cancer, including biopsies, are commonly associated with increased concentrations of CTCs. ${ }^{[13]}$ High CTC numbers are associated with an unfavorable prognosis in many cancers. ${ }^{[13]}$

In 2017, Liu et al. counted CTCs in blood samples taken at baseline $(n=102)$ and later at patients' first clinical evaluation after starting first-line chemotherapy $(n=72)$ in a cohort of women treated for MBC. The result is that their median follow-up was 16.3 months (range: 9.0-31.0 months) and the CTC detection rate was $69.6 \%$ for the baseline samples. ${ }^{[14]}$ They also found that patients with $\leq 2$ CTC/2 $\mathrm{mL}$ at baseline had longer median PFS than did those with $>2$ CTC $/ 2 \mathrm{~mL}$ (17.0 months vs. 8.0 months; $P=0.002$ ), and patients with $\leq 2 \mathrm{CTC} / 2 \mathrm{~mL}$ at both baseline and first clinical evaluation had longest PFS (18.2 months) among all patient groups $(P=0.004) .{ }^{[14]}$ This study confirmed the prognostic value of Pep@MNPs assays for patients with MBC who undergo first-line chemotherapy and offered extra stratification regarding PFS for patients with semantic dementia and a possible indicator for patients at risk for liver metastasis. ${ }^{[14]}$

\section{Achievements of CTC concentration and quantification technologies}

Given that the CTC-based liquid biopsy requires only a small amount of blood sample, such a convenient technique may sound tempting. Nevertheless, a rapid and accurate separation strategy has long remain a burden to hinder the clinical application of CTC-related liquid biopsy. Existing techniques featuring capture, isolation, enumeration, and purification of CTC are more or less defected, ${ }^{[15]}$ which pose a barrier to prevent post-biopsy analysis becoming practical. Multiple technologies have been developed for the capture of CTC, taking advantage of the absence of tumor-specific epitopes in normal blood cells; variations in their physical properties such as size, density, and electromechanical characteristics; or application of high-throughput imaging to unpurified blood cell preparations. ${ }^{[11]}$

Traditionally, CTC collection strategies include two overarching category: positive enrichment and negative enrichment. The positive enrichment was developed earlier and thereby more mature, which featured specific binding between the capturing reagent and the characterized protein site on the surface of a CTC. The two major approaches of positive enrichment are cell filtration and microfluidic chip separation.

\section{Positive enrichment technology (Microfluidics)}

In July 2008, Dr. Maheswaran and fellows first achieved the CTC collection by anti-epithelial cell adhesion molecule (EpCAM) antibody selection. The article "Detection of Mutations in EGFR in Circulating Lung-Cancer Cells" by Maheswaran et al. demonstrated that an exhaustive enrichment of CTCs was possible if fostered by a microfluidic chip. The whole blood sample containing CTCs are treated with EpCAM antibody-tagged magnetic beads. The magnetic beads bound to CTCs are dispersed in and separated from the solution by an external magnetic field in the CTC chip. ${ }^{[16]}$ The CTCs obtained will then be eluted from the magnetic beads with solution containing corresponding enzymes. Although developing this automatic separation apparatus is novel and substantial, a major flaw is that the filtration specificity based on EpCAM affinity are not eligible for those CTCs that do not express EpCAM gene, which result in false negative results. Moreover, some CTCs captured may be activated by the EpCAM antibody that may alter their surface characters, and elution can lead to a loss of CTCs and cell-free ctDNA in post-capture analysis.

Ko et al. (2017) have developed a microchip platform that combines fast, magnetic micropore-based negative immunomagnetic selection $(>10 \mathrm{~mL}$ h-1) with rapid onchip in situ RNA profiling $(>100 \times$ faster than conventional RNA labeling). This integrated chip can isolate both rare circulating cells and cell clusters directly from whole blood and allow individual cells to be profiled for multiple RNA cancer biomarkers, achieving sample-to-answer in less than $1 \mathrm{~h}$ for $10 \mathrm{~mL}$ of whole blood. ${ }^{[17]}$ They show that in a cohort of patient samples $(N=25)$, this device can detect and perform in situ RNA analysis on CTCs in patients with pancreatic cancer, even in those with extremely sparse CTCs ( $<1$ CTC mL-1 of whole blood). ${ }^{[17]}$

\section{Negative enrichment technology}

Given that CTCs usually present in bloodstream and the surfaces of white blood cells and red blood cells are less heterogeneous than CTCs, the negative enrichment was suggested and reported by Gao and colleagues. ${ }^{[18]}$ 
As negative enrichment relies on the depletion of red blood cell and white blood cell via anti-CD45 monoclonal antibody, which is independent of surface properties of CTCs, this approach achieves collecting a broader range of CTCs with high heterogeneity.

\section{NanoVelcro technology}

Taking highlights and drawbacks of previous work into account, in April 2016, Lin et al. from the University of California, Los Angeles, proposed and designed a thermosensitive CTC collecting device called NanoVelcro cell-affinity iChip assay. In the article "Nanovelcro Cellaffinity Assay for Detecting and Characterizing Circulating Tumor Cells," Lin and colleagues revealed that the affinity of binding ligands on a CTC to capturing reagents was subject to temperature. ${ }^{[15]} \mathrm{By}$ switching the external temperature between 37 and $4{ }^{\circ} \mathrm{C}$ followed by enzymatic cleavage, this method ensured a complete retrieving of CTCs in the sample without further contamination of CTCs caused by elution or disrupting their normal viability. The CTC acquired from the sample would then undergo in vivo proliferation in patient-derived xenograft (PDX) mice models. ${ }^{[19]}$

\section{CONCLUSIONS AND PERSPECTIVES}

Considering the high viability and vast availability, CTC liquid biopsy provides a possibility of an economical, quick, and accurate indication of tumor advancement. In particular, CTCs works the best in the early stage of cancer as an auxiliary tool to tissue sector, in which the metastatic tendency may remain unnoticed by traditional biopsy or imaging, and before any mild symptoms promptly grow severe. The obstacles of current CTC studies includes post-collection purification, commercialization of a more efficient counting system with lower detection limit relative to the existing CellSearch system, as well as construction of an extensive personalized CTC library. Coupling a spectroscopy determining the CTC density and integrating it with CTC surface analysis, a pocket-sized chip is likely to be converted into a comprehensive multi-batch process in the future.

\section{Conflicts of Interest}

The authors declare no conflict of interest.

\section{REFERENCES}

1. Ashworth TR. A case of cancer in which cells similar to those in the tumours were seen in the blood after death. Med J Aust 1869;14: 146-7.

2. Nowell PC. The clonal evolution of tumor cell populations. Science 1976; 194: 23-8.
3. Lodish H, Berk A. Cancer. In: McHenry B, editors. Molecular Cell Biology, 7th ed. New York: W. H. Freeman; 2012, pp. 1148-9.

4. Zhang D, Zhao L, Zhou P, Ma H, Huang F, Jin M, et al. Circulating tumor microemboli (CTM) and vimentin+ circulating tumor cells (CTCs) detected by a size-based platform predict worse prognosis in advanced colorectal cancer patients during chemotherapy. Cancer Cell Int 2017; 17: 6 .

5. Kasimir-Bauer S, Hoffmann O, Wallwiener D, Kimmig R, Fehm T. Expression of stem cell and epithelial-mesenchymal transition markers in primary breast cancer patients with circulating tumor cells. Breast Cancer Res 2012; 14: R15.

6. Palmirotta R, Lovero D, Silvestris E, Felici C, Quaresmini D, Cafforio P, et al. Next-generation Sequencing (NGS) Analysis on Single Circulating Tumor Cells (CTCs) with No Need of Whole-genome Amplification (WGA). Cancer Genomics Proteomics 2017; 14: 173-9.

7. Dawson S, Tsui WY, Murtaza M, Biggs H, Rueda OM, Chin S, et al. Analysis of Circulating Tumor DNA to Monitor Metastatic Breast Cancer. N Engl J Med 2013, 368: 1199-209.

8. Cristofanilli M, Budd GT, Ellis MJ, Stopeck A, Matera J, Miller MC, et al. Circulating tumor cells, disease progression, and survival in metastatic breast cancer. N Engl J Med 2004; 351: 781-91.

9. Cohen SJ, Punt CJ, Iannotti N, Saidman BH, Sabbath KD, Gabrail NY, et al. Relationship of circulating tumor cells to tumor response, progression-free survival, and overall survival in patients with metastatic colorectal cancer. J Clin Oncol 2008; 26: 3213-21.

10. de Bono JS, Scher HI, Montgomery RB, Parker C, Miller MC, Tissing $\mathrm{H}$, et al. Circulating tumor cells predict survival benefit from treatment in metastatic castration-resistant prostate cancer. Clin Cancer Res 2008; 14: 6302-9.

11. Aceto N, Bardia A, Miyamoto DT, Donaldson MC, Wittner BS, Spencer JA, et al. Circulating tumor cell clusters are oligoclonal precursors of breast cancer metastasis. Cell 2014; 158: 1110-22.

12. Alva A, Friedlander T, Clark M, Huebner T, Daignault S, Hussain M, et al. Circulating Tumor Cells as Potential Biomarkers in Bladder Cancer. J Urol 2015; 194: 790-8.

13. Martin OA, Anderson RL, Narayan K, MacManus MP. Does the mobilization of circulating tumour cells during cancer therapy cause metastasis? Nat Rev Clin Oncol 2017; 14: 32-44.

14. Liu XR, Shao B, Peng JX, Li HP, Yang YL, Kong WY, et al. Identification of high independent prognostic value of nanotechnology based circulating tumor cell enumeration in first-line chemotherapy for metastatic breast cancer patients. Breast 2017; 32: 119-25.

15. Lin M, Fong A, Chen S, Zhang Y, Chen JF, Do P. Nanovelcro Cell-Affinity Assay for Detecting and Characterizing Circulating Tumor Cells. In: Fan Z, Vitha MF, editors. Circulating Tumor Cells: isolation and analysis. Hoboken: John Wiley \& Sons, Inc.; 2016, pp. 201-26.

16. Maheswaran S, Sequist LV, Nagrath S, Ulkus L, Brannigan B, Collura $\mathrm{CV}$, et al. Detection of Mutations in EGFR in Circulating Lung-Cancer Cells. N Engl J Med 2008, 359: 366-77.

17. Ko J, Bhagwat N, Yee SS, Black T, Redlinger C, Romeo J, et al. A magnetic micropore chip for rapid ( $<1$ hour) unbiased circulating tumor cell isolation and in situ RNA analysis. Lab Chip 2017. [Epub ahead of print]

18. Gao Y, Zhu Y, Zhang Z, Zhang C, Huang X, Yuan Z. Clinical significance of pancreatic circulating tumor cells using combined negative enrichment and immunostaining-fluorescence in situ hybridization. J Exp Clin Cancer Res 2016; 35: 66.

19. Williams ES, Rodriguez-Bravo V, Chippada-Venkata U, De Ia IglesiaVicente J, Gong Y, Galsky M, et al. Generation of Prostate Cancer Patient Derived Xenograft Models from Circulating Tumor Cells. J Vis Exp 2015; 105: 53182.

How to cite this article: Wang $Y$, Zhou $Y, H u Z$. The functions of circulating tumor cells in early diagnosis and surveillance during cancer advancement. J Transl Intern Med 2017; 5: 135-38. 